\title{
Effects of sevoflurane and propofol on the development of pneumonia after esophagectomy: a retrospective cohort study
}

Guo-Hua Zhang ${ }^{*}$ (D) and Wen Wang

\begin{abstract}
Background: Postoperative pneumonia (PP) is one of the common complications following esophagectomy and associated with poor short- and long-term outcomes. Sevoflurane and propofol, which have inflammatory-modulating effects, are common used general anesthetics. This study aimed to compare the effects of anesthesia with sevoflurane and propofol on the development of PP after esophageal surgery for cancer.

Methods: The electronic medical records of patients who underwent elective esophagectomy between July 2013 and July 2016 were reviewed. We conducted univariate and multivariate logistics analysis and propensity score matching analysis to compare the effect of sevoflurane and propofol on the incidence of PP and to identify the risk factors for PP after esophagectomy.

Results: Overall, the incidence of postoperative pneumonia was 9.5\%. There was no significant difference in the rates of PP between sevoflurane group and propofol group either before or after propensity score matching (9.6\% vs $8.0 \%$, $P=0.606 ; 7.7 \%$ vs $6.4 \%, P=0.754$, respectively). Univariate and multivariate analysis revealed that alcohol use (OR 1.513; 95\% Cl 1.062-2.156), surgical procedure (Sweet: referent; Ivor-Lewis: OR 1.993; 95\% Cl 1.190-3.337; Three-incision: OR 1. 878; 95\% Cl 1.296-2.722) and surgeon experience (high-volume: referent; low-volume: OR 1.525; 95\% Cl 1.090-2.135) were significant risk factors of postoperative pneumonia.
\end{abstract}

Conclusions: Sevoflurane did not differ from propofol in terms of affecting the risk of PP development after esophagectomy.

Keywords: Sevoflurane, Propofol, Pneumonia, Esophagectomy

\section{Background}

Postoperative pneumonia (PP) is one of the most frequently observed complications following esophagectomy for cancer and has a potentially negative impact on short- and long-term outcomes [1-3]. The incidence of PP following esophagectomy ranges from 8.7 to $38 \%$ according to many previous studies [2-5]. In recent years, several studies have also been performed to investigate potential risk factors of postoperative pneumonia. Advanced age, greater American Society of

\footnotetext{
*Correspondence: d1974@163.com

Department of Anesthesiology, National Cancer Center/Cancer Hospital, Chinese Academy of Medical Sciences and Peking Union Medical College,

No. 17 Panjiayuannanli Road, Chaoyang District, Beijing 100021, China
}

Anesthesiologists (ASA) physical status, chronic obstructive pulmonary disease (COPD), smoking history, alcohol use, prolonged operative time, preoperative chemoradiotherapy, type of operation, and high intraoperative blood loss were found to be predictors for developing postoperative pneumonia [4, 6-8].

The high rate of postoperative pneumonia may result from surgical trauma and one-lung ventilation (OLV) [9]. The former can elicit the release of proinflammatory cytokines while the latter induce pulmonary inflammatory reactions $[9,10]$. These reactions play an important role in the development of subsequent systemic inflammatory disorders [11, 12]. Although OLV results in alveolar proinflammatory effects, their extent may 
depend on administration of anesthetics [13]. Sevoflurane and propofol are commonly used general anesthetics and have been shown to modulate the inflammatory responses in experimental and clinical studies [14-18]. However, there have been few clinical studies to investigate which anesthetic can be more helpful to reduce PP after esophageal surgery for cancer.

Thus, our retrospective study aimed to (1) compare the effects of sevoflurane-based inhalational and propofol-based total intravenous anesthesia on the incidence of PP and (2) to identify risk factors of PP following esophagectomy.

\section{Methods}

This is a retrospective cohort study. Patients who underwent elective esophageal cancer surgery under general anesthesia between July 2013 and July 2016 in our hospital were identified through our electronic medical recording system.

We excluded patients with missing data or those who were not anesthetized with sevoflurane or propofol. All eligible patients were divided into two groups based on anesthetic method: sevoflurane group and propofol group. Anesthesia was induced with midazolam, propofol, opioids, cisatracurium/rocuronium in all patients and maintained with sevoflurane or propofol combined with opioids in each group, respectively. During twolung ventilation and OLV, tidal volume was set to 6$8 \mathrm{~mL} / \mathrm{kg}$ with positive end expiratory pressure (PEEP) $\left(5-10 \mathrm{~cm} \mathrm{H}_{2} \mathrm{O}\right)$ if necessary and peak inspiratory pressure kept under $35 \mathrm{~cm} \mathrm{H}_{2} \mathrm{O}$. The fraction of inspired oxygen was adjusted to achieve pulse oxygen saturation $>95 \%$ and the respiratory rate set to maintain end-tidal $\mathrm{CO}_{2}$ concentrations between 35 to $45 \mathrm{mmHg}$. Generally, neostigmine $2 \mathrm{mg}$ and atropine $1 \mathrm{mg}$ were injected to reverse the action of muscle relaxants at the end of surgery. Most of the patients were routinely extubated at the end of surgery in the operating theatre and then transferred to the post-anesthesia care unit (PACU). They usually stayed in PACU for at least 30 min and were discharged from PACU after reaching the discharge criteria. Only those who did not meet the extubation standards were transferred to intensive care unit (ICU) for mechanical ventilation. Besides, some patients who underwent reoperation or those who developed severe respiratory dysfunction $\left(\mathrm{PaO}_{2}<60 \mathrm{mmHg}\right.$ and/or $\mathrm{PaCO}_{2}>55 \mathrm{mmHg}$ ) due to various causes in surgical ward after surgery were re-admitted to ICU for ventilator support. Some patients transferred to ICU only received close observation and further treatment without being ventilated. Patient-controlled intravenous analgesia was used in both groups.

All studied variables were obtained by reviewing the electronic medical records and divided into baseline characteristics, intraoperative parameters, and postoperative outcomes. A detailed description of studied variables was listed in Table 1 . The primary outcome was the occurrence of postoperative pneumonia which was suspiciously diagnosed based on clinical features (body temperature $\geq$ $38{ }^{\circ} \mathrm{C}$, leukocyte count $>11.0 \times 10^{9} / \mathrm{L}$ or $<4.0 \times 10^{9} / \mathrm{L}$ and purulent secretions) and confirmed by radiographic findings (infiltrative abnormalities) or tracheal aspirates culture $[1,19]$. Once diagnosed as pneumonia, patients were generally treated with antibiotics, physical therapy (taking deep breath, coughing, chest percussion, atomization inhalation, aspiration of sputum), and if necessary, mechanical ventilation according to hospital protocol. We only included patients with definite PP diagnosis which was indicated explicitly in the electronic medical records.

Data were reported as means \pm standard deviation (SD) or frequency and percentage (n, \%). The Student $\mathrm{t}$ test, Mann-Whitney U test, Chi-square test, or Fisher's exact test was performed for statistical analysis when appropriate. Propensity score matching analysis was also used to allow an unbiased comparison. Patients in propofol group were matched with those in sevoflurane group at a ratio of 1:1 using the nearest neighbor matching with a caliper of 0.001 . Besides, we also conducted univariate and multivariate logistic regression analysis to identify independent risk factors for PP. Variables with a $P<0.1$ in univariate analysis were entered in multivariate logistic regression model. Odd ratios (OR) with 95\% confidence intervals $(\mathrm{CI})$ were calculated. A value of $P<$ 0.05 was considered statistically significant. All statistical analysis were conducted using the SPSS software (Statistical Package for the Social Sciences, version 22.0, SPSS Inc., Chicago, IL).

\section{Results \\ Study population}

In the study period, we identified a total of 2321 patients through our electronic medical records. Of these patients, 662 were excluded from this study according to our exclusion criteria, including 36 patients with missing data and 626 patients anesthetized with anesthetics other than sevoflurane or propofol. Thus, 1659 patients were included in the final analysis. The mean age was $60.7 \pm 8.4$ years old with $83.4 \%(n=1383)$ being male. Among the included 1659 patients, 1571 (94.7\%) received sevoflurane inhalational anesthesia while only 88 (5.3\%) received propofol intravenous anesthesia. Overall, 158 (9.5\%) patients developed postoperative pneumonia. In our cohort of 1659 patients, 92 (5.5\%) were admitted or readmitted to the ICU. Only 38 (2.3\%) patients did not meet the extubation standards, 33 $(2.0 \%)$ were re-intubated for mechanical ventilation and $2(0.1 \%)$ received noninvasive ventilation. 
Table 1 Comparison of preoperative and intraoperative variables before and after propensity score matching between sevoflurane group and propofol group

\begin{tabular}{|c|c|c|c|c|c|c|c|}
\hline \multirow[t]{2}{*}{ Variables } & \multirow{2}{*}{$\begin{array}{l}\text { Total } \\
(n=1659)\end{array}$} & \multicolumn{3}{|l|}{ Before Matching } & \multicolumn{3}{|l|}{ After Matching } \\
\hline & & Group S $(n=1571)$ & Group P $(n=88)$ & $P$-value & Group S $(n=78)$ & Group P $(n=78)$ & $P$-value \\
\hline Age (years) & $60.7 \pm 8.4$ & $60.7 \pm 8.4$ & $61.0 \pm 8.1$ & 0.740 & $60.9 \pm 8.0$ & $60.7 \pm 8.1$ & 0.890 \\
\hline Male & $1383(83.4)$ & $1304(83.0)$ & 79 (89.8) & 0.097 & $65(83.3)$ & $69(88.5)$ & 0.357 \\
\hline BMI $\left(\mathrm{kg} \cdot \mathrm{m}^{-2}\right)$ & $23.6 \pm 3.2$ & $23.7 \pm 3.2$ & $23.3 \pm 3.4$ & 0.251 & $23.2 \pm 3.4$ & $23.2 \pm 3.5$ & 0.926 \\
\hline$A S A \geq I I I$ & 209 (12.6) & $206(13.1)$ & $3(3.4)$ & 0.008 & $2(2.6)$ & $2(2.6)$ & 1.000 \\
\hline Hypertension & $416(25.1)$ & $338(24.7)$ & $28(31.8)$ & 0.134 & $12(15.4)$ & $22(28.2)$ & 0.052 \\
\hline Diabetes mellitus & $117(7.1)$ & $105(6.7)$ & $12(13.6)$ & 0.013 & $6(7.7)$ & $6(7.7)$ & 1.000 \\
\hline Coronary artery disease & $75(4.5)$ & $69(4.4)$ & $6(6.8)$ & 0.286 & $2(2.6)$ & $2(2.6)$ & 1.000 \\
\hline Alcohol use & $972(58.6)$ & $917(58.4)$ & $55(62.5)$ & 0.444 & $43(55.1)$ & $46(59.0)$ & 0.628 \\
\hline Smoking history & $1071(64.6)$ & 1009 (64.2) & $62(70.5)$ & 0.235 & $47(60.3)$ & $55(70.5)$ & 0.178 \\
\hline Preoperative chemoradiotherapy & $130(7.8)$ & $128(8.1)$ & $2(2.3)$ & 0.046 & $3(3.8)$ & $2(2.6)$ & 0.649 \\
\hline \multicolumn{8}{|l|}{ Preoperative Laboratory } \\
\hline $\mathrm{GLU}(\mathrm{mmol} / \mathrm{L})$ & $5.5 \pm 1.0$ & $5.5 \pm 1.0$ & $5.4 \pm 1.0$ & 0.503 & $5.3 \pm 0.7$ & $5.4 \pm 0.9$ & 0.570 \\
\hline $\mathrm{Cr}(\mu \mathrm{mol} / \mathrm{L})$ & $75.9 \pm 14.1$ & $75.8 \pm 14.1$ & $77.4 \pm 13.6$ & 0.319 & $76.7 \pm 12.5$ & $77.1 \pm 13.9$ & 0.832 \\
\hline $\operatorname{ALB}(\mathrm{g} / \mathrm{L})$ & $43.9 \pm 3.3$ & $43.9 \pm 3.3$ & $43.8 \pm 3.3$ & 0.599 & $43.6 \pm 3.4$ & $43.6 \pm 3.2$ & 0.930 \\
\hline Hct (\%) & $42.4 \pm 4.5$ & $42.4 \pm 4.5$ & $42.6 \pm 4.0$ & 0.766 & $42.6 \pm 3.5$ & $42.6 \pm 4.0$ & 0.990 \\
\hline Total fluids (mL) & $2657 \pm 761$ & $2649 \pm 761$ & $2809 \pm 747$ & 0.054 & $2710 \pm 698$ & $2736 \pm 692$ & 0.818 \\
\hline Total crystalloid (mL) & $1896 \pm 615$ & $1881 \pm 613$ & $2160 \pm 593$ & $<0.001$ & $2071 \pm 561$ & $2100 \pm 529$ & 0.736 \\
\hline Total colloid (mL) & $668 \pm 375$ & $672 \pm 380$ & $585 \pm 265$ & 0.004 & $603 \pm 305$ & $577 \pm 268$ & 0.578 \\
\hline Operation time (min) & $249 \pm 95$ & $248 \pm 95$ & $255 \pm 90$ & 0.537 & $262 \pm 88$ & $245 \pm 80$ & 0.212 \\
\hline Intraoperative hypotension ${ }^{a}$ & $275(16.6)$ & $266(16.9)$ & $9(10.2)$ & 0.100 & $8(10.3)$ & $9(11.5)$ & 0.797 \\
\hline Intraoperative hypoxemia ${ }^{\text {b }}$ & $227(13.7)$ & $214(13.6)$ & $13(14.8)$ & 0.760 & $11(14.1)$ & $11(14.1)$ & 1.000 \\
\hline Blood transfusion & 225 (13.6) & $216(13.7)$ & $9(10.2)$ & 0.348 & $5(6.4)$ & $7(9.0)$ & 0.548 \\
\hline Tumor location & & & & 0.002 & & & 0.876 \\
\hline Upper third & $161(9.7)$ & $155(9.9)$ & $6(6.8)$ & & $3(3.8)$ & $5(6.4)$ & \\
\hline Middle third & $729(43.9)$ & $686(43.7)$ & $43(48.9)$ & & $39(50.0)$ & $38(48.7)$ & \\
\hline Lower third & $449(27.1)$ & $415(26.4)$ & $34(38.6)$ & & $32(41.0)$ & $30(38.5)$ & \\
\hline Gastroesophageal junction & $320(19.3)$ & $315(20.1)$ & $5(5.7)$ & & $4(5.1)$ & $5(6.4)$ & \\
\hline Tumor histology & & & & 0.116 & & & 0.585 \\
\hline Adenocarcinoma & $378(22.8)$ & $365(23.2)$ & $13(14.8)$ & & $12(15.4)$ & $13(16.7)$ & \\
\hline Squamous & $1177(70.9)$ & $1106(70.4)$ & $71(80.7)$ & & $60(76.9)$ & $62(79.5)$ & \\
\hline Other & $104(6.3)$ & $100(6.4)$ & $4(4.5)$ & & $6(7.7)$ & $3(3.8)$ & \\
\hline Tumor stage & & & & 0.085 & & & 0.346 \\
\hline I & $302(18.2)$ & $292(18.6)$ & $10(11.4)$ & & $10(12.8)$ & $8(10.3)$ & \\
\hline$\|$ & $486(29.3)$ & $450(28.6)$ & $36(40.9)$ & & $27(34.6)$ & $31(39.7)$ & \\
\hline III & $714(43.0)$ & $677(43.1)$ & $37(42.0)$ & & $31(39.7)$ & $35(44.5)$ & \\
\hline IV & $5(0.3)$ & $5(0.3)$ & $0(0)$ & & $0(0)$ & $0(0)$ & \\
\hline NA & $152(9.2)$ & $147(9.4)$ & $5(5.7)$ & & $10(12.8)$ & $4(5.1)$ & \\
\hline Minimally invasive approach & $321(19.3)$ & $306(19.5)$ & $15(17.0)$ & 0.574 & $14(17.9)$ & $13(16.7)$ & 0.832 \\
\hline Surgical procedure & & & & 0.902 & & & 0.561 \\
\hline Sweet & $1136(68.5)$ & 1077 (68.6) & $59(67.0)$ & & $50(64.1)$ & $55(70.5)$ & \\
\hline Ivor-Lewis & $148(8.9)$ & $139(8.8)$ & $9(10.2)$ & & $11(14.1)$ & $7(9.0)$ & \\
\hline
\end{tabular}


Table 1 Comparison of preoperative and intraoperative variables before and after propensity score matching between sevoflurane group and propofol group (Continued)

\begin{tabular}{|c|c|c|c|c|c|c|c|}
\hline \multirow[t]{2}{*}{ Variables } & \multirow{2}{*}{$\begin{array}{l}\text { Total } \\
(n=1659)\end{array}$} & \multicolumn{3}{|l|}{ Before Matching } & \multicolumn{3}{|l|}{ After Matching } \\
\hline & & Group S $(n=1571)$ & Group P $(n=88)$ & $P$-value & Group S $(n=78)$ & Group P $(n=78)$ & $P$-value \\
\hline Three-incision & $375(22.6)$ & $355(22.6)$ & $20(22.7)$ & & $17(21.8)$ & $16(20.5)$ & \\
\hline Surgeon experience & & & & $<0.001$ & & & 0.872 \\
\hline High-volume ${ }^{c}$ & 1099 (66.2) & $1058(67.3)$ & $41(46.6)$ & & $35(44.9)$ & $36(46.2)$ & \\
\hline Low-volume ${ }^{d}$ & $560(33.8)$ & $513(32.7)$ & $47(53.4)$ & & $43(55.1)$ & $42(53.8)$ & \\
\hline
\end{tabular}

Values are expressed as mean \pm standard deviation or number of patients $(n, \%)$

Abbreviations: $S$ sevoflurane, $P$ propofol, BMI body mass index, ASA American Society of Anesthesiologist, GLU glucose, $C r$ serum creatinine, $A L B$ albumin, $H c t$ hematocrit, NA not available

antraoperative hypotension: lowest systolic blood pressure $<80 \mathrm{mmHg}$ or use of any vasopressor during operation

bIntraoperative hypoxemia: arterial oxygen saturation by pulse oximetry $<90 \%$

'High-volume: $\geq 100$ esophagectomy in three years

dLow-volume: $<100$ esophagectomy in three years

\section{Anesthetic type}

The baseline characteristics and intraoperative parameters of the included patients according to the anesthetic type were presented in Table 1 . Before propensity matching, there was an uneven distribution of confounders between sevoflurane and propofol groups. More patients experienced preoperative chemoradiotherapy $(P=0.046)$ and less had a history of diabetes mellitus $(P=0.013)$ in sevoflurane group compared with propofol group. Besides, patients in the propofol group were infused more crystalloid $(P<0.001)$ and less colloid $(P=0.004)$ than those in sevoflurane group. There were also significant differences in ASA classification $(P=0.008)$, tumor location $(P=0.002)$ and surgeon experience $(P<0.001)$ between two groups. To allow an unbiased comparison, a propensity score matching analysis was conducted to minimize intergroup differences in some cofounders [20]. After propensity matching, 78 patients in propofol group were successfully matched with 78 patients in sevoflurane group with all $P$ values $>0.05$ (Table 1 ), ensuring an even distribution of confounders between two groups.

Table 2 showed comparisons of postoperative clinical outcomes before and after propensity score matching.
Before propensity score matching, 151 (9.6\%) patients in sevoflurane group and $7(8.0 \%)$ in propofol group developed PP. After propensity score matching, the incidences of PP were 7.7 and $6.4 \%$ in sevoflurane group and propofol group, respectively. No statistically significant difference in the incidence of PP was found between sevoflurane and propofol groups either before $(P=0.606)$ or after propensity score matching $(P=$ $0.754)$. Although the average postoperative hospital stay was significantly longer in sevoflurane group compared with propofol group before propensity score matching $(P=0.003)$, it was not significantly different between two groups after propensity score matching $(P=0.307)$. The other postoperative clinical outcomes also showed no difference between two groups either before or after propensity score matching.

\section{Risk factors for PP}

The results of both the univariate and multivariate analysis of the risk factors for PP were summarized in Table 3. Variables with $P$ value $<0.1$ in univariate analysis and our interested factor (anesthetic) were entered in multivariate logistic regression model to identify independent

Table 2 Comparison of postoperative clinical outcomes before and after propensity score matching between sevoflurane group and propofol group

\begin{tabular}{|c|c|c|c|c|c|c|}
\hline \multirow[t]{2}{*}{ Postoperative Outcomes } & \multicolumn{3}{|l|}{ Before Matching } & \multicolumn{3}{|l|}{ After Matching } \\
\hline & Group S $(n=1571)$ & Group P $(n=88)$ & $P$-value & Group S $(n=78)$ & Group P $(n=78)$ & $P$-value \\
\hline Postoperative pneumonia & $151(9.6)$ & $7(8.0)$ & 0.606 & $6(7.7)$ & $5(6.4)$ & 0.754 \\
\hline ICU admission & $88(5.6)$ & $4(4.5)$ & 0.674 & $4(5.1)$ & $2(2.6)$ & 0.405 \\
\hline Reintubation & $33(2.1)$ & $0(0)$ & 0.170 & $2(2.6)$ & $0(0)$ & 0.155 \\
\hline Reoperation & $34(2.2)$ & $0(0)$ & 0.163 & $1(1.3)$ & $0(0)$ & 0.316 \\
\hline Total hospital stay ${ }^{a}$ & $20 \pm 12$ & $19 \pm 7$ & 0.061 & $19 \pm 7$ & $18 \pm 6$ & 0.566 \\
\hline Postoperative hospital stay ${ }^{b}$ & $15 \pm 11$ & $13 \pm 6$ & 0.003 & $13 \pm 5$ & $12 \pm 5$ & 0.307 \\
\hline In-hospital mortality & $3(0.2)$ & $0(0)$ & 0.682 & $0(0)$ & $0(0)$ & - \\
\hline
\end{tabular}

Values are expressed as mean \pm standard deviation or number of patients $(n, \%)$

Abbreviations: $S$ sevoflurane, $P$ propofol, ICU intensive care unit

${ }^{a}$ Total hospital length of stay: period between the admission and discharge day

${ }^{b}$ Postoperative length of stay: period between the day of surgery and the day of discharge 
Table 3 Univariate and Multivariate analyses of factors associated with postoperative pneumonia

\begin{tabular}{|c|c|c|c|c|c|c|}
\hline \multirow[t]{2}{*}{ Characteristics } & \multicolumn{3}{|c|}{ Univariate } & \multicolumn{3}{|c|}{ Multivariate } \\
\hline & $\mathrm{OR}$ & $95 \% \mathrm{Cl}$ & $P$-value & $\mathrm{OR}$ & $95 \% \mathrm{Cl}$ & $P$-value \\
\hline BMl & 0.933 & $0.886-0.983$ & 0.010 & 0.935 & $0.887-0.985$ & 0.012 \\
\hline Alcohol use & 1.592 & $1.122-2.261$ & 0.009 & 1.513 & $1.062-2.156$ & 0.022 \\
\hline Total fluids & 1.000 & $1.000-1.000$ & 0.071 & & & \\
\hline Total crystalloid & 1.000 & $1.000-1.000$ & 0.096 & & & \\
\hline Blood transfusion & 1.570 & $1.026-2.401$ & 0.038 & & & \\
\hline Operation time & 1.003 & $1.001-1.004$ & 0.001 & & & \\
\hline Anesthetic & & & 0.607 & & & \\
\hline Sevoflurane & 1 & Referent & & & & \\
\hline Propofol & 0.813 & $0.369-1.791$ & & & & \\
\hline Tumor location & & & 0.080 & & & \\
\hline Gastroesophageal junction & 1 & Referent & & & & \\
\hline Upper third & 2.128 & $1.109-4.081$ & & & & \\
\hline Middle third & 1.797 & $1.079-2.993$ & & & & \\
\hline Lower third & 1.467 & $0.840-2.561$ & & & & \\
\hline Tumor histology & & & 0.090 & & & \\
\hline Adenocarcinoma & 1 & Referent & & & & \\
\hline Squamous & 1.594 & $1.027-2.475$ & & & & \\
\hline Other & 1.128 & $0.495-2.572$ & & & & \\
\hline Minimally invasive approach & 1.417 & $0.965-2.080$ & 0.076 & & & \\
\hline Surgical procedure & & & $<0.001$ & & & 0.001 \\
\hline Sweet & 1 & Referent & & 1 & Referent & \\
\hline Ivor-Lewis & 2.019 & $1.211-3.367$ & & 1.993 & $1.190-3.337$ & \\
\hline Three-incision & 1.922 & $1.330-2.777$ & & 1.878 & $1.296-2.722$ & \\
\hline Surgeon experience & & & 0.010 & & & 0.039 \\
\hline High-volume $^{\text {a }}$ & 1 & Referent & & 1 & Referent & \\
\hline Low-volume ${ }^{b}$ & 1.550 & $1.111-2.161$ & & 1.525 & $1.090-2.135$ & \\
\hline
\end{tabular}

Abbreviation: $B M I$ body mass index, $O R$ odd ratios, $C l$ confidence intervals

aHigh-volume: $\geq 100$ esophagectomy in three years

bow-volume: $<100$ esophagectomy in three years

risk factors for PP. On multivariate analysis, the independent risk factors for PP were alcohol use (OR 1.513; 95\% CI 1.062-2.156), surgical procedure (Sweet: referent; IvorLewis: OR 1.993; 95\% CI 1.190-3.337; Three-incision: OR 1.878; 95\% CI 1.296-2.722) and surgeon experience (highvolume: referent; low-volume: OR 1.525; 95\% CI 1.0902.135). It was noteworthy that increased body mass index (BMI) appeared to be associated with decreased likelihood of PP (OR 0.935; 95\% CI 0.887-0.985). However, neither univariate nor multivariate analysis showed that propofol was associated with the risk of developing PP.

\section{Discussion}

We conducted this retrospective single-center study to explore the relationship between anesthetic agent and postoperative pneumonia in patients who underwent elective esophageal cancer surgery. Overall, the incidence of PP was 9.6\% in sevoflurane group compared with 8.0\% in propofol group. Neither the entire cohort nor the propensity score-matched cohort showed any statistically significant difference in PP incidence between two groups.

Propofol and sevoflurane are common general anesthetics used in clinical practice. Some clinical researches have investigated the pulmonary inflammatorymodulating effects of sevoflurane and propofol during OLV. Their results suggested that sevoflurane suppressed the local alveolar inflammatory responses and reduced inflammatory mediators release in patients undergoing OLV, resulting in better clinical outcomes in thoracic surgery $[13,21,22]$. A recent meta-analysis by Sun et al. also showed that inhalational anesthetics might be preferable for OLV during thoracic anesthesia due to their protective effects of attenuating inflammatory responses when compared with propofol-based intravenous anesthesia [23]. 
However, previous studies mainly addressed the effects of anesthetics on release of inflammatory markers without showing enough data on postoperative clinical outcomes. In addition, esophagectomy might produce greater systemic stress response and less alveolar injury than pulmonary surgery. To our knowledge, few studies have systematically compared the effects of propofol and sevoflurane on the development of PP after esophageal surgery. In our study, we found that two different anesthesia methods had similar effects on the PP rate in patients undergoing esophagectomy. This finding was in accordance with that of Lee's study, where sevoflurane was not proven to be superior to propofol in lowering postoperative pulmonary complications although the former attenuated increases in inflammatory markers at the end of esophageal operations [9]. It seems that inflammatory-modulating effects of sevoflurane and propofol may not be persistent in the postoperative period [9, 24].

In this study, we also demonstrated that alcohol use, surgical procedure and surgeon experience were independent risk factors of PP while patients with increased BMI appeared to be associated with less likelihood of PP. As BMI of most patients in this study remained within normal range, the effect of BMI on PP should be considered as merely statistical significance, the corresponding clinical relevance may require further investigation. COPD and preoperative pulmonary infections are determining factors in PP development. However, these diseases were rare in our hospital. Only 14 (0.8\%) patients (14 in sevoflurane group and 0 in propofol group, $P=0.374$ ) were diagnosed with COPD according to their medical history and pulmonary function and no patients had pulmonary infections prior to operation. As for patients with preoperative pulmonary infections, they were transferred to general hospitals for treatment till the symptoms and signs were completely improved. Otherwise, they would not actually be admitted for surgery. Our findings as well as previous published results have also indicated that patients' physical status and operation correlative factors affected the development of PP more than anesthetics did after esophagectomy.

Several limitations should be considered when interpreting the results in our study. First, certain confounding factors may affect the results given the retrospective design of our study despite the application of propensity score matching analysis. Secondly, we only recruited the patients with confirmed diagnosis of postoperative pneumonia which was indicated explicitly in our electronic medical records. Thirdly, COPD and preoperative pulmonary infections were not considered as comorbidities in this study as these diseases were rare in our hospital. As a specialized cancer center, most patients in our hospital were in better conditions and of less comorbidity than those in general hospitals. Besides, we were lack of data about those with preoperative pulmonary infections who returned to our hospital for surgery after effective treatment. Fourthly, we did not take into account the quality and the type of analgesia (e.g. different opioids or analgesic schemes) because all patients in our study were given intercostal nerve block and patient-controlled intravenous analgesia, and the surgeons would administrate additional analgesics when patients complained of pain. Moreover, postoperative physical therapy such as chest percussion, sputum suctioning, etc., seemed to interfere with pain assessment. Fifthly, patients' blood samples were not regularly obtained to measure levels of inflammatory markers such as IL-6, IL-8 and IL-10. Although we hypothesized that the anesthetics may alter the postoperative inflammation, there were no data available to suggest postoperative inflammation alteration given the retrospective and observational design of our study. We only compared the effect of anesthetics on the incidence of postoperative pneumonia following esophagectomy. Sixthly, postoperative residual curarization (PORC) was a relevant factor for the development of PP [25]. Although all the extubated patients stayed in PACU for at least $30 \mathrm{~min}$ and were discharged from PACU after reaching the discharge criteria, there may be still a risk of morbidity in PORC after tracheal extubation under the guidance of only clinical judgment. However, train-of-four (TOF) stimulation monitor was not regularly applied in our hospital and we were lack of relevant data.

\section{Conclusions}

Postoperative pneumonia occurred in $9.5 \%$ of patients after esophagectomy in our hospital. Independent predictors of postoperative pneumonia include alcohol use, surgical procedure and surgeon experience. Anesthetic type does not influence the PP risk after esophageal surgery for cancer.

\section{Abbreviations \\ ALB: Albumin; ASA: American Society of Anesthesiologist; BMI: Body mass index; Cl: Confidence intervals; COPD: Chronic obstructive pulmonary disease; Cr: Serum creatinine; GLU: Glucose; Hct: Hematocrit; ICU: Intensive care unit; NA: Not available; OLV: One-lung ventilation; OR: Odd ratios; P: Propofol; PACU: Postanesthesia care unit; PEEP: Positive end expiratory pressure; PORC: Postoperative residual curarization; PP: Postoperative pneumonia; S: Sevoflurane; SD: Standard deviation; TOF: train-of-four}

\section{Acknowledgements}

Not applicable.

\section{Funding}

This work has not been funded by any entity.

\section{Availability of data and materials}

The datasets used or analyzed during the current study are available from the corresponding author upon reasonable request.

Authors' contributions

$\mathrm{GHZ}$ contributed to the conception and design of the study, analysis and interpretation of data, drafting and modifying the manuscript. WW 
contributed to acquisition of data, analysis and interpretation of data, and drafting the article. Both authors read and approved the final manuscript.

\section{Ethics approval and consent to participate}

This study has been performed in accordance with the ethical standards of the Declaration of Helsinki. Our retrospective study was approved by the ethics committee of Cancer Hospital (16-144/1223), and the requirement for informed consent was waived for this study.

\section{Consent for publication}

Not applicable.

\section{Competing interests}

The authors declare that they have no competing interests.

\section{Publisher's Note}

Springer Nature remains neutral with regard to jurisdictional claims in published maps and institutional affiliations.

Received: 19 August 2017 Accepted: 24 November 2017

Published online: 04 December 2017

\section{References}

1. van der Sluis PC, Verhage RJ, van der Horst S, van der Wal WM, Ruurda JP, van Hillegersberg R. A new clinical scoring system to define pneumonia following esophagectomy for cancer. Dig Surg. 2014;31:108-16.

2. Goense L, van Rossum PS, Tromp M, Joore HC, van Dijk D, Kroese AC, et al. Intraoperative and postoperative risk factors for anastomotic leakage and pneumonia after esophagectomy for cancer. Dis Esophagus. 2017;30:1-10.

3. Booka E, Takeuchi H, Nishi T, Matsuda S, Kaburagi T, Fukuda K, et al. The impact of postoperative complications on survivals after Esophagectomy for esophageal cancer. Medicine (Baltimore). 2015;94:e1369.

4. Yoshida N, Watanabe M, Baba Y, Iwagami S, Ishimoto T, Iwatsuki M, et al. Risk factors for pulmonary complications after esophagectomy for esophageal cancer. Surg Today. 2014;44:526-32.

5. Okamura A, Watanabe M, Mine S, Nishida K, Kurogochi T, Imamura Y. Spirometric lung age predicts postoperative pneumonia after Esophagectomy. World J Surg. 2016;40:2412-8

6. Gupta H, Gupta PK, Schuller D, Fang X, Miller WJ, Modrykamien A, et al. Development and validation of a risk calculator for predicting postoperative pneumonia. Mayo Clin Proc. 2013:88:1241-9.

7. Molena D, Mungo B, Stem M, Lidor AO. Incidence and risk factors for respiratory complications in patients undergoing esophagectomy for malignancy: a NSQIP analysis. Semin Thorac Cardiovasc Surg. 2014;26:287-94

8. Ntutumu R, Liu H, Zhen L, YF H, Mou TY, Lin T, et al. Risk factors for pulmonary complications following laparoscopic gastrectomy: a singlecenter study. Medicine (Baltimore). 2016:95:e4567.

9. Lee JJ, Kim GH, Kim JA, Yang M, Ahn HJ, Sim WS, et al. Comparison of pulmonary morbidity using sevoflurane or propofol-remifentanil anesthesia in an Ivor Lewis operation. J Cardiothorac Vasc Anesth. 2012;26:857-62.

10. Tsujimoto H, Takahata R, Nomura S, Kumano I, Matsumoto Y, Yoshida K, et al. Predictive value of pleural and serum interleukin-6 levels for pneumonia and hypo-oxygenations after esophagectomy. J Surg Res. 2013;182:e61-7.

11. D'Journo XB, Michelet P, Marin V, Diesnis I, Blayac D, Doddoli C, et al. An early inflammatory response to oesophagectomy predicts the occurrence of pulmonary complications. Eur J Cardiothorac Surg. 2010;37:1144-51.

12. Bone RC. Toward a theory regarding the pathogenesis of the systemic inflammatory response syndrome: what we do and do not know about cytokine regulation. Crit Care Med. 1996:24:163-72.

13. Schilling $T$, Kozian A, Senturk M, Huth C, Reinhold A, Hedenstierna G, et al. Effects of volatile and intravenous anesthesia on the alveolar and systemic inflammatory response in thoracic surgical patients. Anesthesiology. 2011;115:65-74.

14. Schilling T, Kozian A, Kretzschmar M, Huth C, Welte T, Buhling F, et al. Effects of propofol and desflurane anaesthesia on the alveolar inflammatory response to one-lung ventilation. $\mathrm{Br} J$ Anaesth. 2007:99:368-75.

15. Kawamura T, Kadosaki M, Nara N, Kaise A, Suzuki H, Endo S, et al. Effects of sevoflurane on cytokine balance in patients undergoing coronary artery bypass graft surgery. J Cardiothorac Vasc Anesth. 2006;20:503-8.
16. Song XM, Wang YL, Li JG, Wang CY, Zhou Q, Zhang ZZ, et al. Effects of propofol on pro-inflammatory cytokines and nuclear factor kappaB during polymicrobial sepsis in rats. Mol Biol Rep. 2009;36:2345-51.

17. Sanchez-Conde P, Rodriguez-Lopez JM, Nicolas JL, Lozano FS, Garcia-Criado FJ, Cascajo C, et al. The comparative abilities of propofol and sevoflurane to modulate inflammation and oxidative stress in the kidney after aortic crossclamping. Anesth Analg. 2008;106:371-8. table of contents

18. Hsiao HT, Wu H, Huang PC, Tsai YC, Liu YC. The effect of propofol and sevoflurane on antioxidants and proinflammatory cytokines in a porcine ischemia-reperfusion model. Acta Anaesthesiol Taiwanica. 2016;54:6-10.

19. American Thoracic S. Infectious diseases Society of a. Guidelines for the management of adults with hospital-acquired, ventilator-associated, and healthcare-associated pneumonia. Am J Respir Crit Care Med. 2005;171:388-416.

20. Little RJ, Rubin DB. Causal effects in clinical and epidemiological studies via potential outcomes: concepts and analytical approaches. Annu Rev Public Health. 2000;21:121-45.

21. De Conno E, Steurer MP, Wittlinger M, Zalunardo MP, Weder W, Schneiter D, et al. Anesthetic-induced improvement of the inflammatory response to one-lung ventilation. Anesthesiology. 2009;110:1316-26.

22. Potocnik I, Novak Jankovic V, Sostaric M, Jerin A, Stupnik T, Skitek M, et al. Antiinflammatory effect of sevoflurane in open lung surgery with one-lung ventilation. Croat Med J. 2014:55:628-37.

23. Sun B, Wang J, Bo L, Zang Y, Gu H, Li J, et al. Effects of volatile vs. propofolbased intravenous anesthetics on the alveolar inflammatory responses to one-lung ventilation: a meta-analysis of randomized controlled trials. J Anesth. 2015:29:570-9.

24. Morita M, Yoshida R, Ikeda K, Egashira A, Oki E, Sadanaga N, et al. Acute lung injury following an esophagectomy for esophageal cancer, with special reference to the clinical factors and cytokine levels of peripheral blood and pleural drainage fluid. Dis Esophagus. 2008;21:30-6.

25. Martinez-Ubieto J, Ortega-Lucea S, Pascual-Bellosta A, Arazo-Iglesias I, GilBona J, Jimenez-Bernardó T, et al. Prospective study of residual neuromuscular block and postoperative respiratory complications in patients reversed with neostigmine versus sugammadex. Minerva Anestesiol. 2016;82(7):735-42.

\section{Submit your next manuscript to BioMed Central and we will help you at every step:}

- We accept pre-submission inquiries

- Our selector tool helps you to find the most relevant journal

- We provide round the clock customer support

- Convenient online submission

- Thorough peer review

- Inclusion in PubMed and all major indexing services

- Maximum visibility for your research

Submit your manuscript at www.biomedcentral.com/submit
Biomed Central 